\title{
The long-term safety and efficacy of concomitant Cox maze procedures for atrial fibrillation in patients without mitral valve disease
}

\author{
Niv Ad, MD, ${ }^{\mathrm{a}, \mathrm{b}, \mathrm{c}}$ Sari D. Holmes, PhD, ${ }^{\mathrm{d}}$ Anthony J. Rongione, MD, ${ }^{\mathrm{b}}$ Vinay Badhwar, MD, ${ }^{\mathrm{a}}$ \\ Lawrence Wei, MD, ${ }^{\mathrm{a}}$ Lisa M. Fornaresio, $\mathrm{PhD},{ }^{\mathrm{d}}$ and Paul S. Massimiano, $\mathrm{MD}^{\mathrm{b}}$
}

\section{ABSTRACT}

Objective: Newly published guidelines made the highest level recommendation for surgical treatment for atrial fibrillation. However, the number of patients without a mitral valve procedure with atrial fibrillation who are treated with concomitant surgical ablation is still low (15\%-25\%), because surgeons are reluctant to perform procedures in patients who would not otherwise require left atriotomy. The purpose of this study was to compare the outcomes of concomitant Cox maze with and without mitral valve procedures.

Methods: Patients who underwent concomitant Cox maze procedures were prospectively followed since September 2005. Of the 711 patients, 238 did not receive mitral valve surgery. Propensity score matching was conducted to balance preoperative characteristics between patients with and without mitral valve procedures (164/group after matching).

Results: Before matching, patients in the mitral valve group were younger (65 vs 67 years, $P=.047$ ) and had higher euroSCORE II (European System for Cardiac Operative Risk Evaluation; 3.2\% vs 2.6\%, $P=.002$ ), larger mean left atrial size $(5.3$ vs $4.8 \mathrm{~cm}, P<.001)$, and shorter median atrial fibrillation duration (19 vs 25 months, $P=.064$ ). Early outcomes were similar for the matched groups. Cumulative 5-year freedom from stroke did not differ between matched mitral valve and non-mitral valve groups $(96.1 \%$ vs $96.6 \%$, $P=.667)$. At each time point, the proportion in sinus rhythm off antiarrhythmic medications was similar for the matched groups, including 5 years after surgery $(68 \%$ vs $63 \%, P=.492)$.

Conclusions: The Cox maze procedure is safe and effective with comparable outcomes when performed concomitant to mitral valve or non-mitral valve surgery. Surgeons should base the decision to perform surgical ablation procedures on atrial fibrillation pathophysiology and the benefit to patients, not on the type of concomitant procedure. (J Thorac Cardiovasc Surg 2019;157:1505-14)

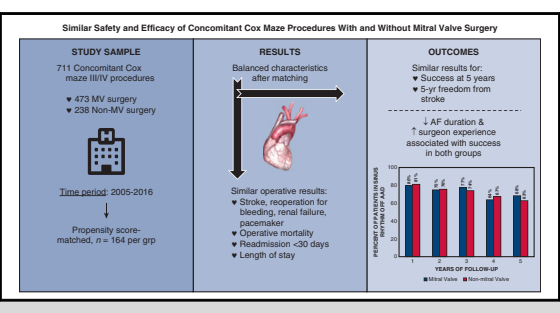

Cox maze procedures with and without MV surgery.

\section{Central Message}

Cox maze procedures should be performed when indicated in patients with $\mathrm{AF}$ and without MV surgery. No excess risk was found for morbidity, arrhythmia recurrence, or strokes.

\section{Perspective}

Despite growing evidence associating surgical ablation for $\mathrm{AF}$ with improved outcomes, surgeons remain reluctant to perform the procedure even in patients with AF undergoing other cardiac surgical procedures. This study shows that the addition of the Cox maze had the same positive impact on outcome in patients with and without MV surgery.

See Commentaries on pages 1515 and pages 1517.

\footnotetext{
From the ${ }^{a}$ Department of Cardiovascular and Thoracic Surgery, West Virginia University, Morgantown, WVa; ${ }^{b}$ Cardiovascular Surgery, Washington Adventist Hospital, Takoma Park, Md; ${ }^{\mathrm{c} I n o v a ~ F a i r f a x ~ H o s p i t a l, ~ F a l l s ~ C h u r c h, ~ V a ; ~ a n d ~}{ }^{\mathrm{d}}$ Heart and Vascular Institute, West Virginia University Medicine, Morgantown, WVa.

Read at the 98th Annual Meeting of The American Association for Thoracic Surgery, San Diego, California, April 28-May 1, 2018.

Received for publication April 25, 2018; revisions received Aug 31, 2018; accepted for publication Sept 19, 2018; available ahead of print Dec 18, 2018.

Address for reprints: Niv Ad, MD, Department of Cardiovascular and Thoracic Surgery, West Virginia University, 1 Medical Center Dr, Morgantown, WV 26506 (E-mail: nivadmd14@gmail.com).

DOI of original article: https://doi.org/10.1016/j.jtcvs.2018.10.134 $0022-5223 / \$ 36.00$

Copyright (c) 2018 by The American Association for Thoracic Surgery https://doi.org/10.1016/j.jtcvs.2018.09.131
}

Concomitant surgical ablation procedures for atrial fibrillation (AF) have been routinely performed successfully since 1987. ${ }^{1}$ However, despite growing evidence associating surgical ablation to improved patient outcome, the adoption and level of proficiency in applying such procedures are variable among surgeons and centers. ${ }^{2-4}$ This phenomenon is unique in the surgical field, and it is evident in the minimal amount of cases and lack of

To view the AATS Annual Meeting Webcast, see the URL next to the webcast thumbnail. 


\section{Abbreviations and Acronyms \\ AATS $=$ American Association for Thoracic Surgery \\ $\mathrm{AF} \quad=$ atrial fibrillation \\ AVR $=$ aortic valve replacement \\ CABG $=$ coronary artery bypass grafting \\ euroSCORE $=$ European System for Cardiac \\ Operative Risk Evaluation \\ LA $\quad=$ left atrial \\ $\mathrm{MV} \quad=$ mitral valve \\ STS $\quad=$ Society of Thoracic Surgeons}

significant post-training proctoring and education to improve the credentialing process in the field. ${ }^{5}$

Publications based on data from North America reveal that only $15 \%$ to $30 \%$ of patients who presented for nonmitral valve (MV) procedures (coronary artery bypass grafting $[\mathrm{CABG}]$ and aortic valve replacement [AVR]) and $\mathrm{AF}$ received surgical ablation. ${ }^{3,6,7}$ In some centers, concomitant surgical ablation is not even considered or performed. The potential explanations for the low percent of ablations performed may be related to the perception that surgical ablation is associated with increased risk, no patient benefit, and the reluctance of surgeons to apply atriotomies in patients who would not otherwise require it. The gap in education regarding the significance of surgical ablation for AF, especially the Cox maze procedure, in improving short- and long-term outcome should be eliminated in years to come.

The primary purpose of this study was to evaluate outcomes in patients who underwent the Cox maze procedure with a non-MV surgical procedure that did not require left atriotomy and compare with similar patients who underwent the Cox maze procedure concomitant with an MV procedure. The secondary aim of this study was to examine the impact of the specific types of concomitant surgery on outcomes within the non-MV group.

\section{MATERIALS AND METHODS}

The sample for this study was composed of patients who underwent concomitant Cox maze procedures between January 2005 and May $2016(N=711)$. Patients were then separated into 2 groups: 473 patients who underwent MV surgery and 238 patients who did not undergo MV surgery. Data on rhythm, medication status, follow-up interventions, and clinical events were captured prospectively according to Heart Rhythm Society guidelines at prespecified time points of 3, 6, 9, 12, 18, and 24 months and then yearly thereafter. These data were merged with the local Society of Thoracic Surgeons (STS) database. ${ }^{8}$ This study was approved by the institutional review board, and a waiver of consent was granted.

\section{Surgical Procedure}

The Cox maze procedure for AF was performed by multiple surgeons using the lesion set described previously. ${ }^{9-12}$ All surgical cases were performed at Inova Fairfax Hospital in Falls Church, Virginia. The decision of whether to perform surgical ablation and the techniques used were left up to the discretion of each individual surgeon. Regardless of the energy source used, the lesion set applied was based on the same principles. Cryoprobes manufactured by both AtriCure, Inc (West Chester, Ohio) and Medtronic (Minneapolis, Minn) were used, and bipolar radiofrequency clamps used were manufactured by AtriCure, Inc. Whenever possible, exit blocks across the pulmonary veins were tested after pulmonary vein isolation for patients in sinus rhythm at the time of surgery. The left atrial (LA) appendage was excised or excluded using an endocardial stitch or AtriClip (AtriCure, Inc) in all cases. Data on the method of LA appendage management technique were available on 436 patients, of whom $62 \%$ had amputation closure ( $\mathrm{MV}=56 \%$, non-MV $=70 \%), 30 \%$ had sutured closure $(\mathrm{MV}=35 \%$, non-MV $=24 \%), 8 \%$ had Atriclip closure $(\mathrm{MV}=8 \%$, non-MV $=6 \%)$, and 1 patient had staple closure $(\mathrm{MV}=0.3 \%$, non- $\mathrm{MV}=0 \%)$.

\section{Statistical Analyses}

All analyses were performed with SPSS Statistics Version 24.0 (IBM Corp, Armonk, NY) or R software version 3.3.2 (R Foundation for Statistical Computing, Vienna, Austria). Continuous variables are presented as mean \pm standard deviation or median (interquartile range), and categorical variables are presented as frequency (percent). Univariate group comparisons were conducted with independent-samples $t$ tests or Mann-Whitney $U$ tests for continuous variables and chi-square tests or Fisher exact tests, as appropriate. Missing values in preoperative left atrium size $(\mathrm{n}=42)$ and duration of AF $(n=45)$ were imputed using the sample means. Observed to expected ratios for operative mortality less than 30 days were calculated using the European System for Cardiac Operative Risk Evaluation (euroSCORE) II as the expected value in patients with and without MV procedures to accommodate for multiple procedures, including the Cox maze. ${ }^{13}$

Propensity score matching was conducted to simulate randomization by balancing preoperative characteristics between patients with and without concomitant MV procedures. ${ }^{14,15}$ The propensity score was estimated with a logistic model using the following covariates selected a priori: age, gender, euroSCORE II (STS risk score is not available for surgical ablation or Cox maze procedures), ejection fraction, diabetes, hypertension, chronic pulmonary disease, peripheral vascular disease, previous cerebrovascular accident, minimally invasive approach, LA size, duration of AF (months), type of $\mathrm{AF}$ (long-standing persistent vs others), preoperative catheter ablation, number of surgical procedures, and elective status. Propensity score matching was conducted between the 2 groups using a caliper of 0.25 propensity score standard deviations. After matching, 164 patients remained in each group and good covariate balance was achieved (Figure 1).

In the full unmatched sample, mixed-model logistic regression was used to identify factors associated with failure during 5 years of follow-up after the Cox maze procedure separately for patients with and without concomitant MV procedures. The purpose of these analyses was to understand whether the factors associated with failure after the Cox maze procedure would be the same for patients with and without MV procedures, rather than to compare the groups on failure, which was conducted within the propensity score-matched analyses. The fixed effects factors in the mixed model were selected a priori and included age, gender, ejection fraction, LA size, duration of AF (years), surgeon experience with surgical ablation (scaled by 10), minimally invasive approach, energy source (cryothermia vs combination), and number of years of follow-up. The random effects factor in the model was the patient. 


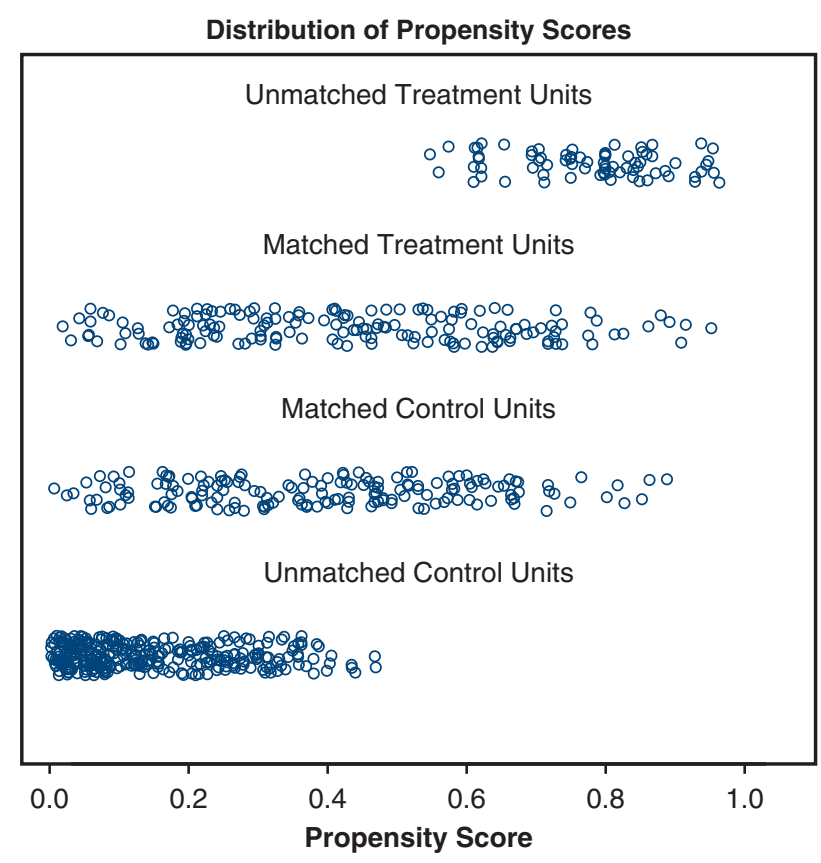

FIGURE 1. Jitter plot showing the distribution of propensity scores for matched and unmatched MV (control units) and non-MV (treatment units) groups, illustrating that after matching, the distribution of propensity scores was balanced between the 2 groups.

\section{RESULTS}

The mean age of this sample was $65.8 \pm 11.0$ years, and $38 \%$ were female. There were significant differences in patient characteristics between the patients who did and did not undergo MV surgery concomitant with the Cox maze procedure (Table 1). Patients with MV surgery were younger $(P=.047)$ and had higher operative risk based on the euroSCORE II $(P=.002)$, a larger LA size $(P<.001)$, and shorter median AF duration before surgery $(P=.064)$. The particular combinations of surgical procedures included with the Cox maze in the non-MV group are presented in Table 2 and were primarily CABG and aortic valve surgery. After matching, patient characteristics were similar and balanced between the MV and non-MV groups (Table 3).

\section{Matched Sample Analyses}

The incidence of perioperative outcomes did not differ for patients with and without concomitant MV procedures, including incidence of stroke $(0.6 \%$ vs $1.2 \%, P>.999)$, prolonged ventilation more than 24 hours $(11 \%$ vs $9 \%$, $P=.582)$, reoperation for bleeding ( $2 \%$ vs $4 \%$, $P=.199)$, renal failure requiring temporary dialysis $(3 \%$ vs $2 \%, P=.723)$, pacemaker implantation $(10 \%$ vs $7 \%$, $P=.385)$, and readmission within 30 days $(13 \%$ vs $16 \%, P=.431)$. The median length of stay in days also did not differ between patients with and without concomitant MV procedures (7 [5-12] vs 7 [5-11], $P=.843$ ).
Operative mortality within 30 days did not differ $(1.8 \%$ vs $1.8 \%, P>.999)$. By using the mean euroSCORE II to obtain expected values, the observed/expected ratios for mortality within 30 days were similar for both the MV and non-MV groups ( 0.42 and 0.41 , respectively).

Analysis of outcomes during follow-up found that cumulative 5-year freedom from embolic stroke was high for all patients and did not differ between those with and without concomitant MV procedures $(96.1 \%$ vs $96.6 \%, P=.667)$ (Figure 2). In regard to rhythm status during follow-up, analyses found that the proportion in sinus rhythm regardless of antiarrhythmic medication was similar for patients with and without MV procedure at 1 year $(88 \%$ vs $91 \%$, $P=.526), 2$ years $(85 \%$ vs $84 \%, P=.943), 3$ years $(83 \%$ vs $83 \%, P=.979), 4$ years $(80 \%$ vs $74 \%$, $P=.321)$, and 5 years after surgery $(80 \%$ vs $72 \%$, $P=.303$ ) (Figure 3, A). The proportion in sinus rhythm off class I/III antiarrhythmic medications was also similar for patients with and without concomitant MV procedures at each time point (Figure 3, B). This result was found at 1 year $(80 \%$ vs $81 \%, P=.781), 2$ years $(75 \%$ vs $76 \%$, $P=.905), 3$ years $(77 \%$ vs $74 \%, P=.579), 4$ years (64\% vs $67 \%, P=.632)$, and 5 years after surgery $(68 \%$ vs $63 \%, P=.492$ ). By 5 years after surgical ablation, more than half of the patients with and without concomitant MV surgery were no longer taking anticoagulants (54\% vs $52 \%, P=.807)$ and the majority of both groups still on anticoagulants were taking them for clinically appropriate reasons (ie, mechanical valve, history of deep vein thrombosis, recurrent atrial arrhythmia).

In the propensity score-matched sample, data on rhythm status were available in 263 patients at 1 year after surgery (19 patients not due, 18 patients died, 6 patients withdrawn, 22 patients with unavailable follow-up), 191 patients at 3 years (66 patients not due, 28 patients died, 17 patients withdrawn, 26 patients with unavailable follow-up), and 136 patients at 5 years after surgery (122 patients not due, 32 patients died, 13 patients withdrawn, 25 patients with unavailable follow-up). Patients who withdrew or with unavailable follow-up did not differ on any patient characteristics from those with available rhythm status at 5 years, except that history of cerebrovascular accident was lower for patients with available rhythm status ( $4 \%$ vs $16 \%, P=.015)$.

\section{Mixed-Model Analyses}

In the interest of maximizing sample size and power to examine the factors associated with atrial arrhythmia recurrence separately by surgery groups, the full nonmatched sample was used to conduct the mixed-model analyses. In these analyses performed separately in the 2 groups, the factors associated with atrial arrhythmia recurrence during follow-up differed slightly by group (Table 4). In the MV group, the factors associated with recurrence were longer preoperative $\mathrm{AF}$ duration $(P=.001)$, fewer cases of surgeon 
TABLE 1. Patient characteristics by surgery group before matching

\begin{tabular}{|c|c|c|c|c|}
\hline Characteristic* & Non-MV group $\mathbf{n}=\mathbf{2 3 8}$ & MV group $n=473$ & $P$ value & Standardized difference \\
\hline Age $(y)$ & $67.0 \pm 10.1$ & $65.3 \pm 11.4$ & .047 & 0.16 \\
\hline Female & $56(24)$ & $212(45)$ & $<.001$ & 0.45 \\
\hline Ejection fraction $(\%)$ & $55.8 \pm 11.6$ & $54.6 \pm 11.0$ & .180 & 0.11 \\
\hline Diabetes mellitus & $63(27)$ & $49(10)$ & $<.001$ & 0.45 \\
\hline Hypertension & $180(76)$ & $289(61)$ & $<.001$ & 0.33 \\
\hline Peripheral vascular disease & $23(10)$ & $27(6)$ & .052 & 0.15 \\
\hline Preoperative creatinine & $1(0.9-1.2)$ & $1(0.8-1.1)$ & .039 & 0.17 \\
\hline Previous cerebrovascular accident & $19(8)$ & $26(6)$ & .199 & 0.08 \\
\hline Chronic pulmonary disease & $53(22)$ & $112(24)$ & .674 & 0.05 \\
\hline Duration of $\mathrm{AF}(\mathrm{y})$ & $2.1(0.3-5.2)$ & $1.6(0.2-4.3)$ & .064 & 0.15 \\
\hline Left atrium diameter $(\mathrm{cm})$ & $4.8 \pm 0.8$ & $5.3 \pm 1.0$ & $<.001$ & 0.55 \\
\hline Nonparoxysmal AF type & $201(85)$ & $405(86)$ & .678 & 0.03 \\
\hline $\mathrm{CHADS}_{2}$ score $\geq 2$ & $117(49)$ & $210(44)$ & .229 & 0.10 \\
\hline $\mathrm{CHA}_{2} \mathrm{DS}_{2}$-VASc score $\geq 2$ & $189(79)$ & $369(78)$ & .668 & 0.02 \\
\hline euroSCORE II (\%) & $2.6(1.6-4.8)$ & $3.2(1.9-6.1)$ & .001 & 0.27 \\
\hline Elective status & $199(84)$ & $400(85)$ & .742 & 0.03 \\
\hline Redo surgery & $16(7)$ & $51(11)$ & .080 & 0.14 \\
\hline Minimally invasive & $4(2)$ & $52(11)$ & $<.001$ & 0.37 \\
\hline Cryothermia only energy source & $129(54)$ & $258(55)$ & .931 & 0.02 \\
\hline Cases of surgeon experience & $161.5(55.8-415.8)$ & $218(70.5-480.5)$ & .098 & 0.13 \\
\hline
\end{tabular}

$M V$, Mitral valve; $A F$, atrial fibrillation; euroSCORE, European System for Cardiac Operative Risk Evaluation. *Data presented as frequency (\%), mean \pm standard deviation, or median (interquartile range).

experience with surgical ablation $(P=.016)$, and combination energy source $(P=.047)$. However, for the non-MV group, the factors associated with recurrence were older age $(P=.001)$, longer AF duration $(P=.008)$, and fewer cases of surgeon experience $(P=.004)$.

TABLE 2. Procedures concomitant to the Cox maze in the non-mitral valve group

\begin{tabular}{lc}
\hline & $\begin{array}{c}\text { Non-MV group } \\
(\mathbf{n}=\mathbf{2 3 8}),(\%)\end{array}$ \\
\hline CABG only & $84(35)$ \\
AVR only & $69(29)$ \\
AVR + CABG & $26(11)$ \\
AVR + other cardiac procedure & $17(7)$ \\
\hline Other cardiac procedure only & $14(6)$ \\
TVR only & $11(5)$ \\
CABG + other cardiac procedure & $8(3)$ \\
TVR + CABG & $2(0.8)$ \\
AVR + TVR & $2(0.8)$ \\
TVR + other cardiac procedure & $2(0.8)$ \\
AVR + CABG + other cardiac procedure & $2(0.8)$ \\
AVR + carotid endarterectomy & $1(0.4)$ \\
\hline
\end{tabular}

$M V$, Mitral valve; $C A B G$, coronary artery bypass graft; $A V R$, aortic valve replacement; $T V R$, tricuspid valve replacement.

\section{Surgery Group Results}

In the full sample of patients who did not have an MV procedure, new perioperative pacemaker implantation occurred in 3 patients (4\%) with CABG as the only concomitant procedure and 2 patients $(3 \%)$ with AVR as the only concomitant procedure. Rhythm results in the full sample of patients who did not have an MV procedure found that the proportion in sinus rhythm regardless of antiarrhythmic medications for patients with aortic valve surgery as the only concomitant procedure was $90 \%$ at 1 year, $81 \%$ at 2 years, $90 \%$ at 3 years, $79 \%$ at 4 years, and $81 \%$ at 5 years after surgery. The proportion in sinus rhythm off antiarrhythmic medications for this group of patients was $82 \%$ at 1 year, $75 \%$ at 2 years, $79 \%$ at 3 years, $70 \%$ at 4 years, and $71 \%$ at 5 years after surgery. For patients with sole concomitant aortic valve procedures, anticoagulation use at 5 years after surgery was $45 \%$, there were no strokes during follow-up, follow-up cardioversions occurred in $13 \%$ of patients, and there were no follow-up catheter ablations.

Likewise, the proportion in sinus rhythm regardless of antiarrhythmic medications for patients with CABG as the only concomitant procedure was $92 \%$ at 1 year, $87 \%$ at 2 years, $87 \%$ at 3 years, $64 \%$ at 4 years, and $69 \%$ at 5 years after surgery. The proportion in sinus rhythm off antiarrhythmic medications for this group of patients was $81 \%$ 
TABLE 3. Patient characteristics by surgery group after matching

\begin{tabular}{|c|c|c|c|c|}
\hline Characteristic* & Non-MV group $n=164$ & MV group $n=164$ & $P$ value & Standardized difference \\
\hline Age $(y)$ & $66.6 \pm 10.3$ & $66.1 \pm 11.0$ & .690 & 0.05 \\
\hline Female & $50(31)$ & $53(32)$ & .721 & 0.02 \\
\hline Ejection fraction $(\%)$ & $54.8 \pm 12.7$ & $54.6 \pm 11.2$ & .858 & 0.02 \\
\hline Diabetes & $31(19)$ & $28(17)$ & .666 & 0.05 \\
\hline Hypertension & $112(68)$ & $114(70)$ & .811 & 0.04 \\
\hline Peripheral vascular disease & $15(9)$ & $15(9)$ & $>.999$ & 0 \\
\hline Preoperative creatinine & $1(0.8-1.2)$ & $1(0.8-1.2)$ & .984 & 0.002 \\
\hline Previous cerebrovascular accident & $11(7)$ & $10(6)$ & .822 & 0.04 \\
\hline Chronic pulmonary disease & $35(21)$ & $37(23)$ & .790 & 0.05 \\
\hline Duration of $\mathrm{AF}(\mathrm{y})$ & $1.9(0.3-4.7)$ & $1.8(0.3-4.1)$ & .581 & 0.06 \\
\hline Left atrium size $(\mathrm{cm})$ & $4.9 \pm 0.8$ & $5.0 \pm 0.8$ & .519 & 0.13 \\
\hline Nonparoxysmal AF type & $139(85)$ & $138(84)$ & .879 & 0.03 \\
\hline $\mathrm{CHADS}_{2}$ score $\geq 2$ & $68(42)$ & $83(51)$ & .097 & 0.18 \\
\hline $\mathrm{CHA}_{2} \mathrm{DS}_{2}$-VASc score $\geq 2$ & 127 (77) & $132(81)$ & .498 & 0.10 \\
\hline euroSCORE II ( $\%)$ & $2.7(1.8-5.4)$ & $3.2(1.8-5.5)$ & .683 & 0.04 \\
\hline Elective status & $138(84)$ & $139(85)$ & .879 & 0.03 \\
\hline Redo surgery & $15(9)$ & $15(9)$ & $>.999$ & 0 \\
\hline Minimally invasive & $4(2)$ & $6(4)$ & .521 & 0.12 \\
\hline Cryothermia only energy source & $89(54)$ & $77(47)$ & .185 & 0.14 \\
\hline Cases of surgeon experience & $177.5(42.5-398.3)$ & $126.5(54-448.8)$ & .754 & 0.03 \\
\hline
\end{tabular}

$M V$, Mitral valve; $A F$, atrial fibrillation; euroSCORE, European System for Cardiac Operative Risk Evaluation. *Data presented as frequency ( $\%)$, mean \pm standard deviation, or median (interquartile range).

at 1 year, $81 \%$ at 2 years, $80 \%$ at 3 years, $55 \%$ at 4 years, and $56 \%$ at 5 years after surgery. For patients with sole concomitant $\mathrm{CABG}$, anticoagulation use at 5 years after surgery was $43 \%$, incidence of stroke was $5 \%(n=4)$, followup cardioversions occurred in $25 \%$ of patients, and followup catheter ablation procedures occurred in $6 \%$ of patients.

Patients in the non-MV group with all other combinations of concomitant procedures $(n=85)$ also experienced an acceptable proportion of patients in sinus rhythm regardless of antiarrhythmic medications, including 1 year $(91 \%)$, 2 years $(80 \%), 3$ years $(80 \%)$, 4 years $(80 \%)$, and 5 years after surgery $(70 \%)$. The proportion in sinus rhythm off antiarrhythmic medications for this group of patients was $83 \%$ at 1 year, $69 \%$ at 2 years, $70 \%$ at 3 years, $73 \%$ at 4 years, and $62 \%$ at 5 years after surgery. For these patients, use of anticoagulation at 5 years after surgery was $53 \%$, incidence of stroke was $2 \%(\mathrm{n}=2)$, follow-up cardioversions occurred in $20 \%$ of patients, and follow-up catheter ablation procedures occurred in $11 \%$ of patients.

Examining rhythm status by surgeon found that the range of patients in the non-MV group with CABG as the only concomitant procedure who returned to sinus rhythm off antiarrhythmic medications was $80 \%$ to $100 \%$ across 6 surgeons at 1 year, $0 \%$ to $100 \%$ across 5 surgeons at 2 years, $0 \%$ to $100 \%$ across 5 surgeons at 3 years, $0 \%$ to $100 \%$ across 5 surgeons at 4 years, and $0 \%$ to $100 \%$ across 5 surgeons at 5 years. For the patients in the non-MV group with aortic valve surgery as the only concomitant procedure, these ranges were $50 \%$ to $100 \%$ across 5 surgeons at 1 year, $0 \%$ to $100 \%$ across 5 surgeons at 2 years, $0 \%$ to $100 \%$ across 4 surgeons at 3 years, $0 \%$ to $100 \%$ across 4 surgeons at 4 years, and $0 \%$ to $100 \%$ across 4 surgeons at 5 years.

\section{DISCUSSION}

This study was focused on patients with clinically significant AF who underwent a non-MV concomitant surgical procedure. The results of this study found that patients who receive a non-MV surgery at the same time as a Cox maze procedure had no excess risk for perioperative morbidities, arrhythmia recurrences, or follow-up strokes compared with patients with MV procedures (Figure 4). Additionally, in both groups the factors that were most associated with recurrences during the first 5 years after the Cox maze procedure were duration of $\mathrm{AF}$ and surgeon experience.

The level of evidence associated with concomitant surgical ablation for AF has improved significantly compared with 10 to 15 years ago. This fact is well communicated through the recent set of guidelines from several associations and societies. ${ }^{2,16,17}$ The general theme of all the guideline documents is that concomitant surgical ablation for $\mathrm{AF}$ is effective across all types of procedures, safe, and associated with improved 30-day survival. ${ }^{2,16,17}$ 


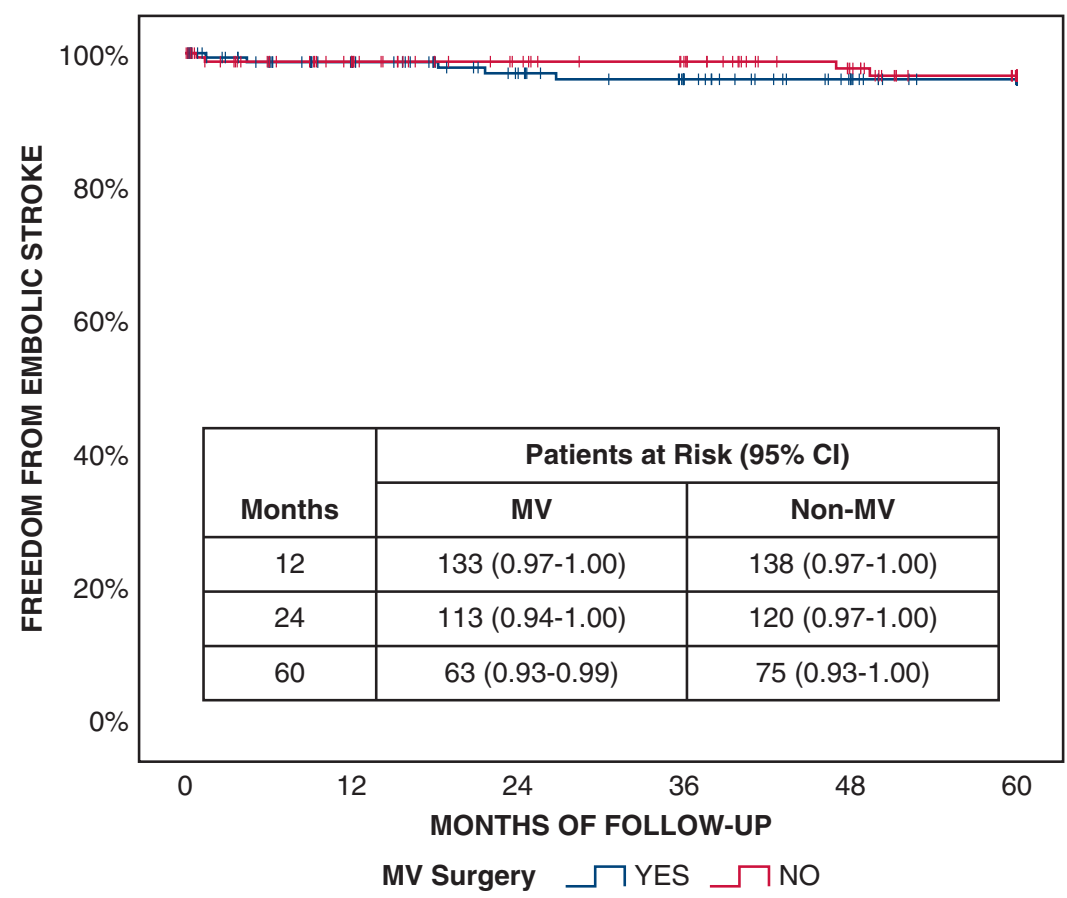

FIGURE 2. Similar 5-year cumulative freedom from embolic stroke in propensity score-matched patients who received Cox maze with and without concomitant MV procedures (log rank $=0.19, P=.667)$. $C I$, Confidence interval; $M V$, mitral valve.

Despite the well-established data and results demonstrating patient benefits, the level of performance of surgical ablation for $\mathrm{AF}$ is variable among centers and surgeons, a unique situation in the surgical field. This fact is especially concerning for concomitant surgical ablation procedures in cases that do not involve the MV and left atriotomy. Different reports from the STS revealed that only $17 \%$ to $30 \%$ of the patients in the non-MV group who presented with $\mathrm{AF}$ would have surgical ablation for AF in the United States. 3,7

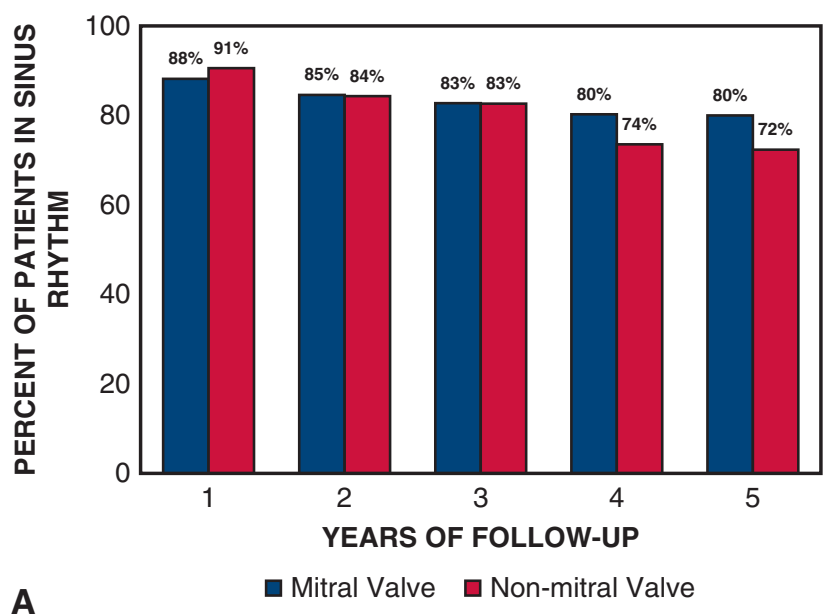

In this study, we sought to evaluate the safety and efficacy of surgical ablation in patients in the non-MV group. There are clear differences between the groups in operative risk, with the MV group having a slightly higher risk based on the euroSCORE II. In regard to traditional predictors for higher recurrence of atrial arrhythmia after surgical ablation, before matching the groups were similar on the type and duration of AF. However, patients in the non-MV group were older and had smaller mean LA size. After propensity score matching, the groups were balanced on surgical risk,

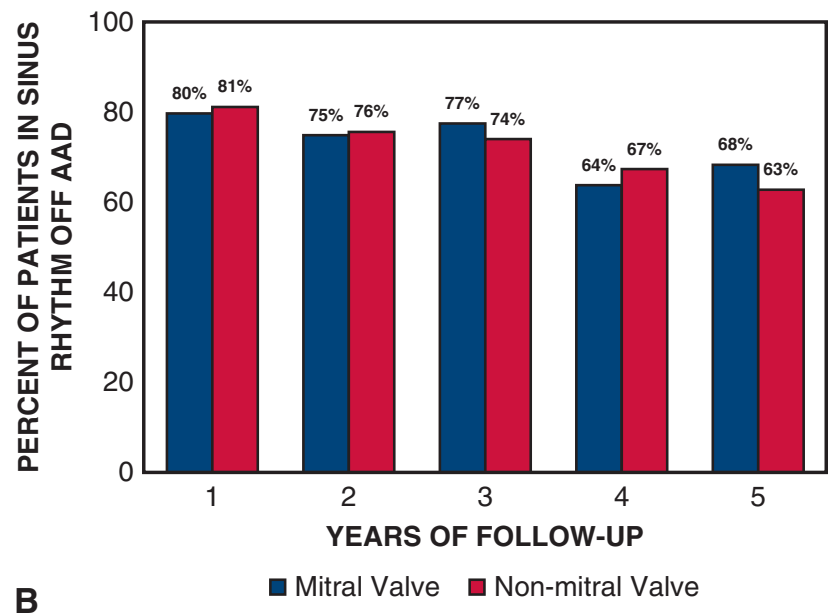

FIGURE 3. Similar proportion of patients who received Cox maze in sinus rhythm (A) and sinus rhythm off class I/III antiarrhythmic medications during follow-up (B) for patients with and without concomitant MV procedures. $A A D$, Antiarrhythmic drug. 
TABLE 4. Mixed-model logistic regression model examining factors associated with atrial arrhythmia recurrence during 5 years after surgical ablation separately for mitral valve and non-mitral valve groups

\begin{tabular}{lcc}
\hline \multicolumn{1}{c}{ Non-MV group $(\mathbf{n}=\mathbf{2 3 8})$} & OR $(\mathbf{9 5} \% \mathbf{C I})$ & $\boldsymbol{P}$ value \\
\hline Age & $1.05(1.02-1.09)$ & .001 \\
Female & $1.36(0.68-2.69)$ & .384 \\
\hline Ejection fraction & $0.98(0.95-1.003)$ & .077 \\
\hline LA size $(\mathrm{cm})$ & $1.31(0.95-1.84)$ & .126 \\
\hline Duration of AF (y) & $1.10(1.03-1.17)$ & .007 \\
\hline Surgeon experience (scaled by 10 cases) & $0.98(0.97-0.996)$ & .014 \\
\hline Minimally invasive approach & $0.99(0.12-8.31)$ & .993 \\
Combination energy source & $1.47(0.83-2.64)$ & .189 \\
\hline Years of follow-up & $1.35(1.21-1.51)$ & $<.001$ \\
\hline \multicolumn{1}{c}{ MV group $(\mathbf{n}=\mathbf{4 7 3})$} & OR (95\% CI) & $\boldsymbol{P}$ value \\
\hline Age & $1.01(0.99-1.03)$ & .270 \\
\hline Female & $1.06(0.68-1.65)$ & .787 \\
\hline Ejection fraction & $0.99(0.97-1.01)$ & .399 \\
\hline LA size (cm) & $1.08(0.86-1.35)$ & .499 \\
\hline Duration of AF (y) & $1.08(1.03-1.12)$ & .001 \\
\hline Surgeon experience (scaled by 10 cases) & $0.99(0.98-0.999)$ & .048 \\
\hline Minimally invasive approach & $0.89(0.40-1.87)$ & .761 \\
\hline Combination energy source & $1.61(1.02-2.53)$ & .039 \\
\hline Years of follow-up & $1.18(1.09-1.29)$ & $<.001$ \\
\hline
\end{tabular}

$M V$, Mitral valve; $O R$, odds ratio; $C I$, confidence interval; $L A$, left atrial; $A F$, atrial fibrillation.

comorbidities, and variables associated with increased risk for failure of surgical ablation.

Perioperative morbidity associated with the procedure was shown to be similar with no significant differences between the groups and the reported results for such procedures that do not include surgical ablation for AF. ${ }^{6,18,19}$ In previous studies, we demonstrated that the addition of surgical ablation to any open procedure and across different risk groups was not associated with an increased risk. ${ }^{6,20}$ Our group also showed in several studies, based on the STS and Medicare data, that surgical ablation in patients undergoing $\mathrm{CABG}$ or MV surgery is not associated with increased surgical risk, but both early and late survival benefits were documented. ${ }^{7,21,22}$ In this study, the observed/expected ratio for both groups using the euroSCORE II revealed no difference in risk for mortality by adding a Cox maze procedure to MV or nonMV indexed procedure(s). A common question and challenge that surgeons are facing is what is the potential impact of the Cox maze procedure on patient outcomes when added to an AVR or CABG. The study also demonstrated an acceptable morbidity across all surgical groups. These findings should be considered by surgeons and referring physicians when patients with clinically significant $\mathrm{AF}$ are referred to surgery. It is also important to notice that the incidence of pacemaker implantation in this study resembles what was reported by the STS and our previous publications. ${ }^{23}$ Of special interest should be the low rate of perioperative pacemaker implantation in patients who required AVR or CABG only, which was the majority of the non-MV group. These rates are lower than reported in other studies in particular when all patients underwent a full Cox maze procedure. ${ }^{24,25}$ In some patients who may require surgical ablation for AF concomitantly with a non-MV procedure, the decision of whether to perform the ablation procedure and which type of procedure should be performed require more discussion. On the basis of our experience, patients with a small left atrium may benefit from LA only ablation if they also have short duration of $\mathrm{AF}$ and paroxysmal $\mathrm{AF}^{26}$ The other group of patients to examine are those with a longer duration of AF. In these patients, the long-term efficacy of the Cox maze procedure is reduced and an appropriate discussion with the patients should take place before the procedure. Partial ablation in these cases is not recommended. ${ }^{16,27}$

Furthermore, this study examined the potential differences in efficacy of the Cox maze procedure in MV and non-MV cases. The main findings demonstrate similar short- and long-term efficacy off and on class I/III antiarrhythmic drugs. The same result was found with short- and long-term freedom from embolic strokes, with more than $96 \%$ freedom from stroke for both groups and more than half of the patients off anticoagulation at 5 years. A subgroup analysis of patients in the non-MV group, including those with AVR or $\mathrm{CABG}$ as the sole concomitant procedure, revealed high safety and efficacy 5 years after surgery. These findings should encourage surgeons to address AF accordingly even if a biatrial lesion set is required. The pathophysiology of AF should play a role in the decision regarding the appropriate lesion set and not the type of concomitant surgery or ablation technology available. This statement is well supported in the STS and American Association for Thoracic Surgery (AATS) guidelines. ${ }^{2,21,28}$

The recent AATS guidelines recommended and highlighted the importance of appropriate training and proctoring for surgical ablation to improve surgeon understanding and proficiency with AF treatments. ${ }^{2}$ In previous publications, we have addressed the importance of surgeon experience and knowledge in the decision of whether to perform surgical ablation and the results of such procedures. ${ }^{4,29}$ The mixed-model analysis showed that surgeon experience at the time of performing the surgical ablation procedure is an important predictor for failure during 5 years of followup. Surgeon experience and expertise may have a direct impact on the success of the procedure. One of the most remarkable examples is the results from the CURE-AF IDE trial. ${ }^{30}$ In that study, the same lesion set and same ablation devices were used, and yet the success rate at 9 months 
Similar Safety and Efficacy of Concomitant Cox Maze Procedures With and Without Mitral Valve Surgery

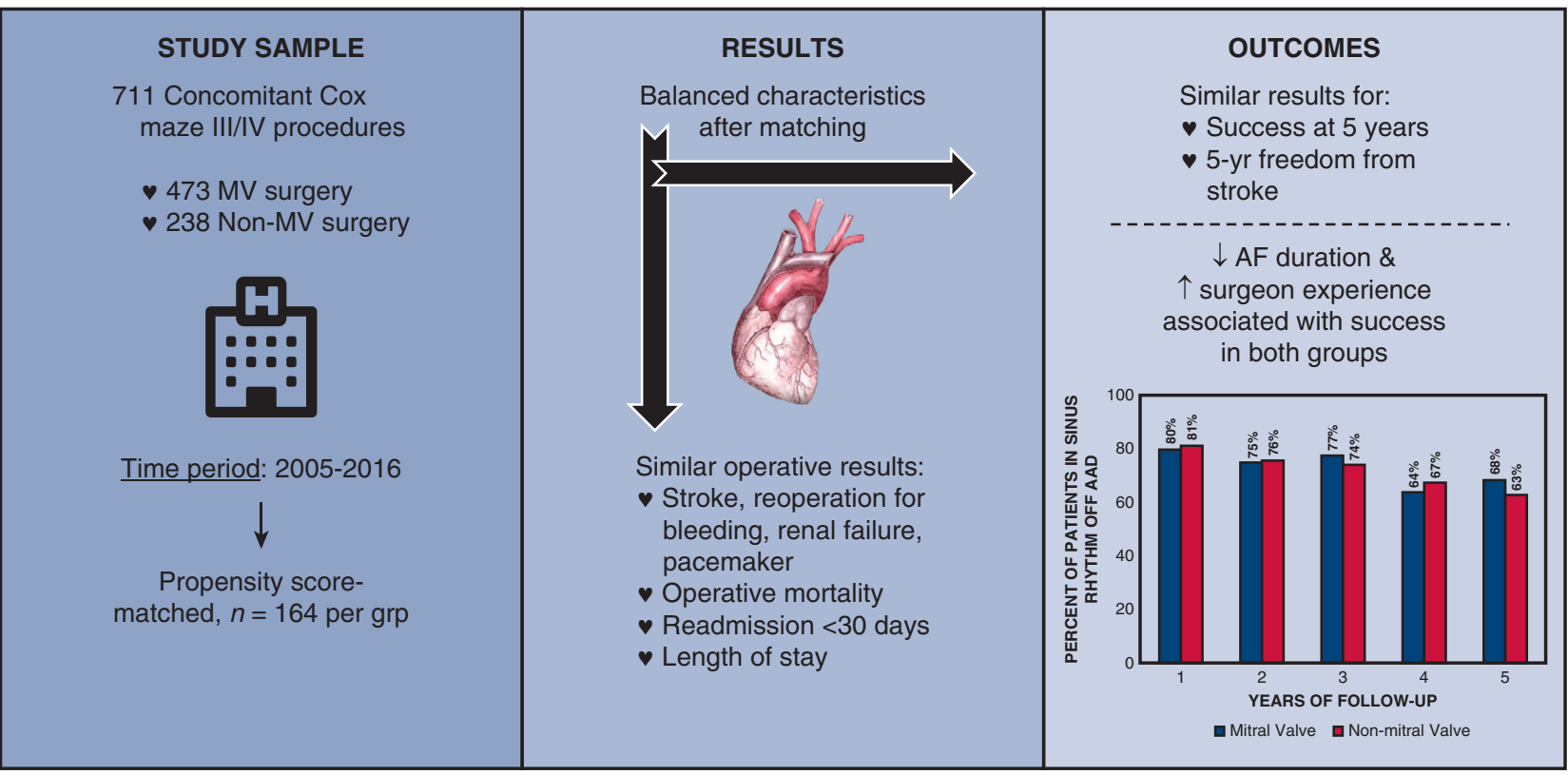

FIGURE 4. Graphical abstract demonstrating that the results of this study found similar safety and efficacy of concomitant Cox maze procedures with and without MV surgery. $A F$, Atrial fibrillation; $M V$, mitral valve.

varied between $33 \%$ and $100 \%$. Interestingly, our current study had similar findings with notable differences between different operators on success rate. In both groups, duration of $\mathrm{AF}$ was found to be significantly associated with atrial arrhythmia recurrence. Duration of AF is another important and consistent predictor in long-term failures and should be readily communicated to referring physicians to facilitate earlier referral for surgery when possible. ${ }^{31}$

There are several important messages to take away from this study. It is clearly demonstrated that the Cox maze procedure was not associated with increased morbidity in patients who did not otherwise require left atriotomy. The study also demonstrated similar long-term success and freedom from embolic strokes in both groups. Once again, we demonstrated that surgeon training and education are fundamental aspects in improving surgical ablation results as recommended in the recent AATS guidelines. ${ }^{2}$ This study was not designed to address the differences between lesion sets that can further change the way surgical ablation is performed, especially in the non-MV cases. Appropriate patient selection may result in excellent outcomes even with a limited lesion set. ${ }^{26}$

\section{Study Limitations}

A limitation of this study was the occurrence of missing data. In addition to missing values on patient characteristics and detailed operative techniques, not all patients had rhythm status available for each time point. Our follow-up program attempted to stay in regular contact with patients after surgical ablation, but losses to follow-up or lack of documentation for physician visits occurred at each time point. Nevertheless, these findings represent the results of a rigorous and detailed prospective follow-up program for patients after surgical ablation for AF.

\section{CONCLUSIONS}

The results of this study indicate that patients who received a non-MV surgery at the same time as a Cox maze procedure had no excess risk for perioperative outcomes, arrhythmia recurrences, or follow-up strokes. Additionally, the factors that were most associated with recurrences during the first 5 years after the Cox maze procedure were duration of $\mathrm{AF}$ and surgeon experience for patients with and without concomitant MV procedures. These findings should encourage surgeons to address comorbid $\mathrm{AF}$ in patients undergoing cardiac surgery, even when a left atriotomy is not otherwise required during surgery.

\section{Webcast}

You can watch a Webcast of this AATS meeting presentation by going to: https://aats.blob.core.windows.net/ media/18Apr29/20ABC $\% 202$.Adult $\% 20$ Cardiac $\% 20$ SS \%20(PM)/S51\%20-\%20Part\%202/S51_5.mp4.

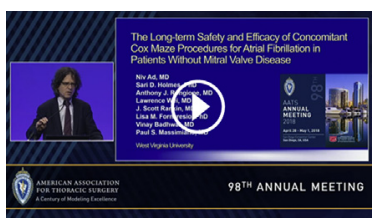




\section{Conflict of Interest Statement}

Ad is a consultant for Medtronic, a member of the speaker's bureau for AtriCure, proctor and member of the speaker's bureau for LivaNova, on the advisory board for Nido Surgical, and co-owner of Left Atrial Appendage Occlusion, LLC. Rongione is a proctor for Medtronic. All other authors have nothing to disclose with regard to commercial support.

All surgical cases were performed at Inova Fairfax Hospital in Falls Church, Virginia. Data were collected and maintained by Deborah Lamont, BSN, RN, and Graciela Pritchard, BS, at Inova Fairfax Hospital in Falls Church, Virginia. The authors thank their colleagues for their contributions.

\section{References}

1. Weimar T, Schena S, Bailey MS, Maniar HS, Schuessler RB, Cox JL, et al. The Cox maze procedure for lone atrial fibrillation: a single-center experience over 2 decades. Circ Arrhythm Electrophysiol. 2012;5:8-14.

2. Ad N, Damiano RJ Jr, Badhwar V, Calkins H, La Meir M, Nitta T, et al. Expert consensus guidelines: examining surgical ablation for atrial fibrillation. J Thorac Cardiovasc Surg. 2017;153:1330-54.

3. Ad N, Suri RM, Gammie JS, Sheng S, O'Brien SM, Henry L. Surgical ablation of atrial fibrillation trends and outcomes in North America. J Thorac Cardiovasc Surg. 2012;144:1051-60.

4. Ad N, Henry L, Hunt S, Holmes SD. Impact of clinical presentation and surgeon experience on the decision to perform surgical ablation. Ann Thorac Surg. 2013; 96:763-9.

5. American Board of Thoracic Surgery Web site. Chicago, IL: The American Board of Thoracic Surgery, Inc; 2018. Available at: https://www.abts.org/. Accessed August 31, 2018

6. Ad N, Henry L, Hunt S, Holmes SD. Do we increase the operative risk by adding the Cox maze III procedure to aortic valve replacement and coronary artery bypass surgery? J Thorac Cardiovasc Surg. 2012;143:936-44.

7. Rankin JS, Lerner DJ, Braid-Forbes MJ, Ferguson MA, Badhwar V. One-year mortality and costs associated with surgical ablation for atrial fibrillation concomitant to coronary artery bypass grafting. Eur J Cardiothorac Surg. 2017;52:471-7.

8. Society of Thoracic Surgeons. The STS surgeon composite technical appendix. February 2015. Available at: http://www.sts.org/sites/default/files/documents/ STS-SurgeonCompositeTechnicalAppendix-UpdatedFeb2015.pdf. Accessed August 31, 2018.

9. Cox JL. The surgical treatment of atrial fibrillation. IV. Surgical technique. J Thorac Cardiovasc Surg. 1991;101:584-92.

10. Cox JL, Schuessler RB, D'Agostino HJJ, Stone CM, Chang BC, Cain ME, et al. The surgical treatment of atrial fibrillation. III. Development of a definitive surgical procedure. J Thorac Cardiovasc Surg. 1991;101:569-83.

11. Cox JL, Ad N. New surgical and catheter-based modifications of the maze procedure. Semin Thorac Cardiovasc Surg. 2000;12:68-73.

12. Damiano RJ Jr, Bailey M. The Cox maze IV procedure for lone atrial fibrillation. Multimed Man Cardiothorac Surg. 2007;2007(723). mmcts.2007.002758.

13. Society of Thoracic Surgeons. Online STS risk calculator. The Society of Thoracic Surgeons. Available at: http://riskcalc.sts.org/stswebriskcalc/ \#/calculate. Accessed August 31, 2018.

14. Ho D, Imai K, King G, Stuart E. Matching as nonparametric preprocessing for reducing model dependence in parametric causal inference. Polit Anal. 2007; 15:199-236.

15. Ho D, Imai K, King G, Stuart E. MatchIt: nonparametric preprocessing for parametric causal inference. J Stat Softw. 2011;42:1-28.

16. Badhwar V, Rankin JS, Damiano RJ Jr, Gillinov AM, Bakaeen FG, Edgerton JR, et al. The Society of Thoracic Surgeons 2017 clinical practice guidelines for the surgical treatment of atrial fibrillation. Ann Thorac Surg. 2017;103:329-41.

17. Calkins H, Hindricks G, Cappato R, Kim YH, Saad EB, Aguinaga L, et al. 2017 HRS/EHRA/ECAS/APHRS/SOLAECE expert consensus statement on catheter and surgical ablation of atrial fibrillation. Heart Rhythm. 2017;14:e275-444.

18. Jacobs JP, Shahian DM, D'Agostino RS, Jacobs ML, Kozower BD, Badhwar V, et al. The Society of Thoracic Surgeons National Database 2017 annual report. Ann Thorac Surg. 2017;104:1774-81.
19. Lee R, McCarthy PM, Wang EC, Vaduganathan M, Kruse J, Malaisrie SC, et al Midterm survival in patients treated for atrial fibrillation: a propensity-matched comparison to patients without a history of atrial fibrillation. J Thorac Cardiovasc Surg. 2012;143:1341-51.

20. Ad N, Henry LL, Holmes SD, Hunt SL. The impact of surgical ablation for atrial fibrillation in high-risk patients. Ann Thorac Surg. 2012;93:1897-904.

21. Badhwar V, Rankin JS, Ad N, Grau-Sepulveda M, Damiano RJ, Gillinov AM, et al. Surgical ablation of atrial fibrillation in the United States: trends and propensity matched outcomes. Ann Thorac Surg. 2017;104:493-500.

22. Rankin JS, Grau-Sepulveda MV, Ad N, Damiano RJ Jr, Gillinov AM Brennan JM, et al. Associations between surgical ablation and operative mortality after mitral valve procedures. Ann Thorac Surg. 2018;105:1790-6.

23. Ad N, Holmes SD, Ali R, Pritchard G, Lamont D. A single center's experience with pacemaker implantation after the Cox maze procedure for atrial fibrillation. J Thorac Cardiovasc Surg. 2017;154:139-46.

24. Gillinov AM, Gelijns AC, Parides MK, DeRose JJ Jr, Moskowitz AJ, Voisine P, et al. Surgical ablation of atrial fibrillation during mitral-valve surgery. $N$ Engl $J$ Med. 2015;372:1399-409.

25. Philpott JM, Zemlin CW, Cox JL, Stirling M, Mack M, Hooker RL, et al. The ABLATE trial: safety and efficacy of Cox maze-IV using a bipolar radiofrequency ablation system. Ann Thorac Surg. 2015;100:1541-8.

26. Ad N, Holmes SD, Lamont D, Shuman DJ. Left-sided surgical ablation for patients with atrial fibrillation who are undergoing concomitant cardiac surgical procedures. Ann Thorac Surg. 2017;103:58-65.

27. Ad N, Holmes SD, Shuman DJ, Pritchard G. Impact of atrial fibrillation duration on the success of first-time concomitant Cox maze procedures. Ann Thorac Surg. 2015;100:1613-9.

28. Ad N, Holmes SD, Rongione AJ, Massimiano PS, Fornaresio LM. Does surgical ablation energy source affect long-term success of the concomitant Cox maze procedure? Ann Thorac Surg. 2017;104:29-35.

29. Ad N, Holmes SD. Early stable sinus rhythm associated with greater success 5 years after surgical ablation. Ann Thorac Surg. 2018;105:1370-6.

30. Damiano RJ Jr, Badhwar V, Acker MA, Veeragandham RS, Kress DC, Robertson JO, et al. The CURE-AF trial: a prospective, multicenter trial of irrigated radiofrequency ablation for the treatment of persistent atrial fibrillation during concomitant cardiac surgery. Heart Rhythm. 2014;11:39-45.

31. Ad N, Holmes SD, Massimiano PS, Rongione AJ, Fornaresio LM. Long-term outcome following concomitant mitral valve surgery and Cox maze procedure for atrial fibrillation. J Thorac Cardiovasc Surg. 2018;155:983-94.

Key Words: atrial fibrillation, surgical ablation, mitral valve surgery

\section{Discussion}

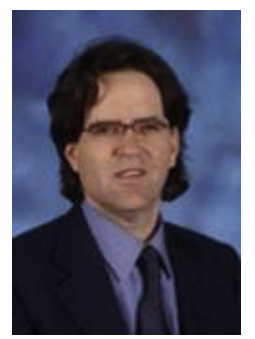

Niv Ad

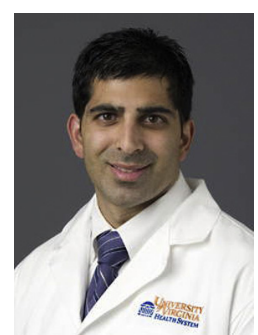

Dr G. Ailawadi (Charlottesville, Va). I congratulate Dr Ad and colleagues for not just this report but for pursuing excellence in surgical AF treatment for many years. An estimated $30 \%$ of patients who require cardiac surgery present to us in AF. Concomitant AF ablation at the time of cardiac surgery remains underused despite a growing body of evidence 
demonstrating improvements in AF burden. In fact, in the state of Virginia, concomitant AF ablation at the time of mitral surgery remains at approximately $50 \%$, still very much underused.

Dr Ad and colleagues have now compared both short- and long-term outcomes of the Cox maze procedure in patients undergoing a concomitant MV operation and a non-MV operation. This study adds to the literature, with more signal, that AF ablation is beneficial, even in patients without MV disease, but still falls short in a number of ways like many studies in this field, which lacks standardization of energy source, lesion set, type of AF, and patient selection.

This series includes patients over a long study period, although the time frame was not clear, and during that time frame there have been different energy sources and different lesions, and the follow-up methods during the study period have evolved. What is the denominator for the data we don't have? How were patients selected? You have 6 surgeons, each with different practices. What is the denominator of the patients who presented with AF who did not receive AF ablation? Do we have any insight as to the patient selection?

Dr Ad. We published these data before. So to avoid repetition, it's not here. To be specific, we never used any energy but bipolar and cryo as one set of energy sources versus cryo alone. There are no variations in the lesion set. It's all Cox maze 3 , by the book, or Cox maze 4 , if you want to call it. So there is no variation there.

The reason for evolution in surgeon experience, and this is why I said it counts from case number 1 to number 50 or more, and we published these data, that the break-even point where you don't see any difference in the evolution and the result is more than 50 cases. I think the data are solid. And patient selection and penetration in our own practice started, and we all publish it, from approximately $30 \%$ in 2004 to approximately $57 \%$ of all-comers in 2016 , more or less. So it grew, but it's not perfect. In some surgeons, the penetration is approximately $20 \%$, and in others, it's more than $90 \%$.

Dr Ailawadi. I will get back to that in the final question, but that's helpful. I was surprised to see, and I know you didn't have time to discuss this in the presentation, that more than $50 \%$ of patients were still on anticoagulation at the time of follow-up, because avoidance of anticoagulation is often the driving goal for patients who present and ask us to perform an ablation. With so many surgeons, what is the practice pattern of anticoagulation in this group? Do you advocate or not advocate for patients remaining on or stopping anticoagulation given that your data suggest that many of them remain out of AF in sinus rhythm?
Dr Ad. I think it's a good observation, but I disagree it's high. I actually think it's a high percentage of patients without anticoagulation, because these patients have a lot of comorbidities. Even if you look at the initial study that I was involved with as a fellow with Dr Cox back in 2000, presented I think even here at the American Association for Thoracic Surgery. Approximately $25 \%$ of the patients discharged from cardiac surgery after surgical ablation will require anticoagulation, otherwise, deep vein thrombosis, pulmonary emboli, mechanical valves, and so forth.

The general practice is to stop anticoagulation after 6 months, and it's not per the surgeon-specific preference. It's the way our program used to be set up and worked with the cardiologists. The reasons not to stop anticoagulation were usually associated with other problems or recurrence of AF. It's consistent, and we published it in many articles that approximately $2 \%$ to $5 \%$ of patients long term are not being followed according to our anticoagulation guidelines.

I want to stress to everybody that we don't do this surgery to stop anticoagulation; we don't promise it to the patients. As a matter of fact, it's not supported by the evidence. We all know the data, and we need to work to get this very carefully, but we also have to be careful to stop anticoagulation in some patients. So I think $50 \%$ is pretty high.

Dr Ailawadi. One more question related to patient selection. In your practice now, who do you think is not a good candidate? As you are presenting this to the general cardiac surgery community, in whom do you think they should avoid performing an open left atriotomy Cox maze?

Dr Ad. Well, I think in general, surgical ablation, I put it all in one, and as Marc alluded to earlier, I do perform an LA only and pulmonary vein isolation only in some patients. But that's an excellent question. In my own practice, basically I don't recommend surgical ablation for AF in patients in whom I don't really think this is going to add anything to them, in other words, patients with other comorbidities, life expectancy is relatively short, and it's very complex surgery. Otherwise, sometimes we would just exclude the appendage and I wouldn't do anything.

But a more important variable is we always look at the right ventricular function. If the right ventricular function is impaired, especially to a moderate to severe degree, I would be reluctant to perform a maze procedure in any patient, because I don't want to extend bypass time to be a minute longer. So those are the 2 basically major groups of patients I wouldn't touch. 